\title{
An Approach to Modify Standard Servo
}

\author{
Yanyan Ji, Jiayu Yang and Jiaqi Wu \\ Computer Science and Technology Programme, United International College, P.R. China
}

\begin{abstract}
The usage of infinite-rotate servos has been pervasive in 3D robot assembling. Hardware companies have been providing expensive infinite-rotate servos for years. But considering limited fund of individual 3D-printed robot project, existing infinite-rotate servos in market are too expensive. This paper introduces a new approach but not easy way to modify non-infinite rotate servos into infinite rotate servos by cutting off the rotation limiting block inside servo and by leading out potentiometer originally inside servo. The result is efficient since the modification save lots time and stable performance for the servos. As for the cost around $90 \%$ cost can be saved. The approach which mentioned in this paper can be used and implemented in different fields; it can be suitable for the cases once the infinite rotate servos are in needed.
\end{abstract}

Keywords-servo; $3 D$ robot; infinite rotate servo; potentiometer

\section{INTRODUCTION}

This paper is based on 3D printed robot project, in this project, we are going to design the movement amounted on 3D printed robot and controlled with servos and smart sensors which implemented by Arduino platform. During the design part, we find a problem, the servo which we used will only rotated from 0 to 180 degree, it is not suitable for the expectation movement, then we spend a lot time to redesign and change the standard servo to be continuous servo. Some of you might asked why we did not use the continuous servos at the first time, the reason is since the specific size which are made by the 3D printed machine and according to the toque we can't find any suitable continuous servos. As mentioned the approached to modify is not easy, the methodology will be given as the second part.

\section{METHODOLOGY}

In our approach, we are using a servo of model HS-805bb+ from Hitek. Before modifying servo, a functional and stable operation environment is required. In our approach, we used an electric iron of 50 watt combined with a hot air heating gun of 700 watt provided by Atten. Higher power hot air heating gun or electric iron is also qualified. Tin suction pump is highly recommended for separating the PCB with motor inside servo when splitting the solder joint. In our case, the W0617 pump from Waynn's Tools is used. Hand saws are required for cutting off the limitation bar. But a better way in removing limitation bar is to use a electric grinder with circular saw blade. We used the QL-800 electric grinder from and set of saw blades Qilan Company. Tweezers are required for handling some small part of servo. Any small tweezer will do. In order to make cable joint connect firmly after soldered, hot glue gun is required. We used the SLT-9A glue gun from Slide any standard glue gun will med the demand. Screwdrivers, soldering tin are also required.

\section{A. Remove the Limitation Bar off}

The main approach to limit servo rotation is to place a plastic bar on the gear inside gear box of servo. So to modify a servo to infinite-rotate, we firstly have to cut the limitation bar off. Three steps are required before cutting.

- First, separate the lid of the servo by screwing out screws of the lid. The printed circuit board (PCB) should be under the lid. Warm up electric iron and hot air heating gun for later use.

- Secondly, take a record of the gear box before taking out any gear. This step should not be skipping as many servos have different gear box and the picture ensures to re-assemble correctly.

- Third step is to identify the limitation block on one of the gears. In our case, HS805BB servo, the limitation block is on the largest gear which directly connected outside (see the Figure I).

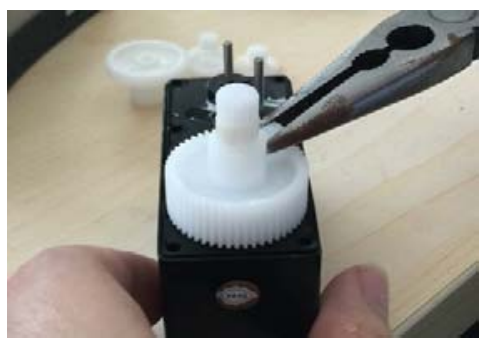

FIGURE I. LIMITATION BLOCK

The final step is to remove the block using hand saw or electric grinder. When using a electric grinder, a bench vise is highly recommended for holding the gear firmly and providing better accuracy and convenience. Make sure the block be cut off without any ragged edge on the gear as any coarse edge can hinder the rotation of gears.

After cutting off limitation block on gear, the gear box should be rotate freely. But other than the gear box, the potentiometer inside servo cans also limited the rotation. Pervasively, potentiometer in market can only rotate less than 360 degree. And nearly all potentiometer inside servo are in this type. So to archive infinite rotation, we have to deal with the potentiometer. Normally we still have the need of identifying and controlling the rotation of infinite-rotation servo in practice, so the potent meter can't be simply removed. If the aim of modification doesn't include further controlling the rotation degree precisely, simply remove the potentiometer 
or replace it with a fixed resistor is recommended. Otherwise, the potentiometer should be separate out from the servo and connected with some parts that the model infinite-rotation servo drives.

\section{B. Take Potentiometer off}

For the two ways mentioned, taking the potentiometer out the servo is both required. Typically the potent meter is placed under the gear box, and also under the PCB. But some servo in market has the potentiometer just under the lid, which is easy to take off. So to move out the potentiometer under PCB and gear box, the PCB must be separate. As motor and PCB are soldered together, we use hot air heating gun and tin suction pump to separate the solder joint. An safe step for beginner is to turn on the hot air heating gun and set it to minimum wind speed and 350 degrees centigrade. Aim at the solder joint and observe the tin on it carefully. Wobble a little to make the heating more balanced and holding the tin suction pump preparing for sucking away the melted tin. Once the tin on the joint become liquid, suck it away quickly. Repeat these steps until the joint become separated (see the Figure II).

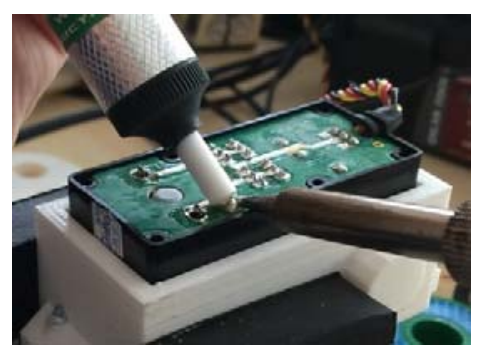

FIGURE II. REMOVING JOINT

After all soldered joints are separated, the PCB should be moved off easily. The motor and potentiometer will be seen. The potentiometer should also be connected to the other side of the PCB and the soldered joint should also be separated. Before separating the joint, take notes of how the wire connected. Use the same way to separate the joints. And the potentiometer should be taken out easily.

\section{Analyze the Different Requirement of Previous Two Steps}

$>$ For modifying that doesn't require precise rotation control or implement the rotation control on software level, replace the potentiometer with a fixed resistor is recommended. Normally the servo will rotate according to the value of resistance provided by potentiometer. When it arrives at the given position, the value of resistance of the potentiometer should be exactly the same with the related value of the position. But for a potentiometer that has been separated out, say, doesn't rotate with the gear, the servo will never reach the correct resistance value. So the servo will rotate forever unless the controller set the position value back to equal to the resistance value of the potentiometer. By replacing the potentiometer with a fixed resistor, the value that servo stops rotating become fixed. The resistance value of the fixed resistor should be half of the total resistance value of the potentiometer and thus set a position less or greater than the medium position will make servo rotate to different side and set a position of exactly the medium position will stop it.

$>$ For modifying that requires further precise position control, the potentiometer will be used outside the servo for detecting the position. In our case, the model infinite-rotate servo drives the arm of the robot to move. The arm rotates from 0 degree to 180 degree but, for every degree, the servo should rotate for degrees to drive it. So to convert the rotation of arm to the rotation of servo, we simply connect the potentiometer to the arm. We set position for the arm and servo drives the arm to rotate. When arrives at the requested position, the potentiometer will inform the servo that it arrives the correct position.

So to use the potentiometer outside the servo, the wire connecting potentiometer and PCB should be prolonging. We use a normal wire to do it (see the Figure III).

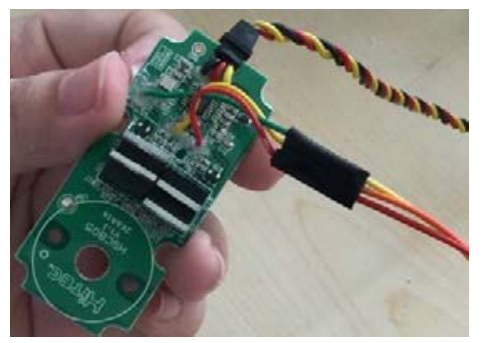

FIGURE III. PROLONG

After dealing with the potentiometer, the servo will be fully capable of infinite rotation. To reassemble the servo, we have to reconnect the solder joints of PCB, reassemble the gear box and then close the lid. Rejoining the solder joints contain four steps. Firstly, warm up the electric iron. Place the PCB to the right place and, when electric iron ready, melt some tin on each joint to reconnect them. Close the lid after checked the joints been connected firmly.

\section{RESULT}

The servos modified by our approach works well in many places. In particular, when driving the arm of our robot, it can fetch the arm's rotating degree by the potentiometer wired out and rotate according to it. It rotates freely and infinitely, which perfectly meets our need.

\section{CONCLUSION}

We presented a solution to lower the cost in building robots with servos that are required to rotate infinitely. To use cheaper servo meeting the requirement of infinite rotating, we introduced an approach to modify normal servo to rotate infinitely. In particular, two different requirements are met with some different way in the approach. And the outcome is proved to be functional.

\section{ACKNOWLEDGEMENT}

This Paper is supported by Research Grant of Beijing Normal University-Hong Kong Baptist University United 
International College G201623.

\section{REFERENCE}

[1] Y. Ji, "How to control DC motor using ZX," IEEE, 2012.

[2] M. B. Nazir, Electro Hydraulic Servo System, VDM vERLAG Dr. MullerAKtiengesellschaft\&Co, 1988.

[3] J. F. A.,. W. B. J.Weldon Bellvile, "Continuous servo motor integration of the electrical activity of the brain and its application to the control of cyclopropane anesthesia," Elsevier INC, pp. 317-320, 1954.

[4] K.V.Viswanatha\&M.Satyam, "Electronic Potentiometer," IETE Journal of Research, pp. 742-743, 2015.

[5] P.E.WELLSTEAD\&P.ZANKER, "Servo self-tuners," International Jouranl of Control, vol. 30, pp. 27-36, 2007.

[6] Y. J. \&. D. M. Hvidsten, "DC Motor Speed Control," in MACE, 2012.

[7] H. D. L. Kendall F Grestch, "Development of novel 3D-printed robotic prosthetic for transradial amputees," in IEEE, 2015.

[8] E. K. Mukhtar, "Control of 3D printed Robot hand actuated by artificial muscles," in SAI Intelligent Systems Conference.

[9] M. Korayem, "Path Planning algorithm in wheeled movile manipulators based on motion of arms," Mechanical Science and Technology, pp. 1753-1763. 